\title{
Especialistas médicos: brechas, realidad, futuro y necesidad de un esfuerzo nacional mancomunado
}

\begin{abstract}
Corporación Nacional Autónoma de Certificación de Especialidades Médicas (CONACEM): José Manuel López Moreno, Presidente; Miriam Alvo Abodovsky, Vicepresidenta, Asociación Facultades de Medicina de Chile; Luis Hervé Allamand, Secretario Ejecutivo; Hernán Chamorro Balart, Tesorero, Colegio Médico de Chile AG.; Carlos Saieh Andonie, Secretario, Sociedad Chilena de Pediatría; Jorge Aguiló Martínez, Asociación Facultades de Medicina de Chile; Gloria López Stewart, Sociedad Médica de Chile; Osvaldo Llanos López, Sociedad de Cirujanos de Chile; Lorenzo Naranjo Tapia, Colegio Médico de Chile AG.; Mauricio Osorio Ulloa, Colegio Médico de Chile AG., Enrique Oyarzún Ebensperger, Sociedad Chilena de Obstetricia y Ginecología; Emilio Roessler Bonzi, Asociación de Facultades de Medicina de Chile; Vicente Valdivieso Dávila, Academia de Medicina del Instituto de Chile; Luis Velozo Papez, Colegio Médico de Chile AG.
\end{abstract}

\section{RESUMEN}

Este documento es una declaración oficial de la "Corporación Nacional Autónoma de Certificación de Especialidades Médicas" (CONACEM), sobre la brecha conocida entre la oferta y la necesidad de especialistas médicos en Chile. La definición de médico especialista exige un alto estándar calidad profesional. La certificación como especialista se puede obtener por tres vías: a) haber sido aprobado por un programa de especialización universitario acreditado; b) el cumplimiento de los requisitos de un programa de formación práctica de cinco años; c) ser aprobado por un programa de formación de otro país, validado en Chile. Este artículo analiza los factores que aumentan la necesidad de especialistas: deficiente capacidad de resolución de la atención primaria, maladistribución geográfica, distribución desigual entre los sistemas médicos públicos y privados, cambios demográficos en el país, cambios sociológicos de la población y déficit en la gestión de la salud. Este artículo también considera los elementos que limitan la oferta de especialistas (deficiencias de los programas de capacitación, baja contribución del sector privado, financiación estatal insuficiente, y remuneraciones no competitivas) y discute las posibles soluciones. La formación en práctica, que representa el camino elegido por el $35 \%$ de los especialistas, debería reforzarse y mejorarse en el tiempo, manteniendo o incrementando calidad.

\section{PALABRAS CLAVE: Médicos especialistas, certificación de especialidad, déficit de especialistas}

\section{SUMMARY}

This document is an official statement from "Corporación Nacional Autónoma de Certificación de Especialidades Médicas" (CONACEM), about the well-known gap between offer and need of medical specialists in Chile. A medical specialist is defined under high standard medical quality. Certification as a specialist can be obtained in three ways: a) being approved by a reputable university specialization program; b) fulfilling the requirements of a five year practical training program; c) be approved by a training program from another country which was validated in Chile. This article discusses factors which are increasing the need for 
specialists: no primary care operative medicine, geographic mal distribution, unequal distribution between public and private medical systems, demographic changes in the country, sociological changes of the population and deficits in health management. This article also considers elements that are limiting the supply of specialists, and discusses possible solutions (shortcomings of training programs, low contribution from the private sector, still insufficient state funding, and uncompetitive remunerations). Training in practice, which accounts for $35 \%$ of specialists, should be enhanced and improved on while maintaining the quality.

\section{KEY WORDS: Medical specialists, medical specialties certification, specialist's deficit}

\section{INTRODUCCION}

La Corporación Nacional Autónoma de Certificación de Especialidades Médicas (CONACEM) es una Corporación de Derecho Privado que, desde su fundación en 1984, ha tenido la responsabilidad de la certificación de 12.500 especialistas médicos y la definición de especialidades médicas. Frente al tema de la falta de especialistas la Corporación ha decidido manifestar su opinión en un afán de aportar en la solución de este acuciante problema.

La demanda por especialistas médicos es tema de gran actualidad. Respecto de la falencia de ellos se habla de brechas, representando la distancia entre lo que se necesita y lo que se oferta; el resultado es percibido por la población como claramente insatisfactorio, y la autoridad así también lo reconoce.

Un análisis juicioso y ponderado del problema requiere la concurrencia de dos elementos: a) Definir el concepto de especialista como la condición que posee quien domina el conocimiento, diagnóstico y tratamiento de las enfermedades de una disciplina reconocida como especialidad. Además, el especialista debe empeñarse por difundir su disciplina, hacerla progresar y eventualmente desarrollar o al menos entender la investigación propia de su campo. En esta definición se basa la calidad del especialista, y el respeto a la fe pública cuando el médico que se presenta como tal lo es en propiedad. b) Cuantificar ajustadamente el problema para diseñar, aplicar estrategias de solución y evaluar respuestas consonantes con las particularidades de la realidad chilena.

Las vías para alcanzar la calidad de especialista en el contexto ya señalado son tres: a) aprobar un programa universitario de formación acreditado en Chile; b) completar los requisitos y la evaluación después de 5 años de práctica de la especialidad; c) haber tenido formación equivalente a la chilena en el extranjero y aprobado las evaluaciones pertinentes.

El presente documento se refiere fundamentalmente a las dos primeras vías de formación; sin embargo, respecto de los especialistas formados en el extranjero, ya sea chilenos o de otras nacionalidades, asistimos actualmente a un claro incremento de su participación porcentual, superando ya el $6 \%$ del total de certificaciones hechas.

Para la consideración ordenada del tema deben analizarse los puntos sustantivos que atañen a las dos vertientes de la brecha, la de la oferta y la de la demanda de especialistas.

\section{ANÁLISIS DE CAUSALIDAD}

\section{Razones del aumento en la demanda por espe-} cialistas:

a) El trasfondo permanente y de gran impacto, es la deficiente capacidad resolutiva de la atención primaria, que desvía hacia las instancias secundarias o terciarias gran parte de su carga. Esta es una limitante permanente y principal que invalida substantivamente los esfuerzos y políticas focalizadas en las especialidades.

b) Inadmisible distribución geográfica de los especialistas, concentrados en Santiago y las dos o tres ciudades más pobladas del país. Una distancia de $200 \mathrm{kms}$ puede significar, en cuanto a la medicina a la cual se accede, a grandes diferencias en su modernidad.

c) Inequitativa distribución de especialistas entre el sector público y el privado. El $50 \%$ de los médicos atiende al sector privado con dos millones de personas, y el otro $50 \%$ a los quince millones restantes. Esta mala distribución se repite y potencia, haciéndose dramática, en las ciudades en falencia por el factor geográfico.

d) Cambios demográficos de la población que aumentan los requerimientos de especialidades (ej. geriatría) o hacen menos urgentes a otras.

e) Demandas de la población relacionadas con la calidad y seguridad del acto médico (ej. anestesia simultánea en dos pabellones a cargo de un solo anestesiólogo). El factor ético relacionado es insoslayable para los responsables de la atención: profesionales, instituciones, y autoridad.

f) Características sociológicas y culturales de nuestra población que recurre directamente al especialista que estima como el adecuado a su dolencia.

g) Empoderamiento social de la población que exige atención de especialistas en situaciones que no lo ameritan.

h) Reducida permanencia de los especialistas 
jóvenes en sus lugares de destinación por falta de estímulos y de una política que fomente el arraigo.

i) Ineficiencia de las atenciones por dificultades de gestión (retardo de exámenes, dación de horas, falta de equipamiento tecnológico, etc.).

j) Tendencia a dar respuesta numérica a las demandas, con menor énfasis en sus resultados.

2. Razones que influyen negativamente en la potencial oferta de especialistas:

a) Falencias y limitaciones cuantitativas y cualitativas de los programas de formación universitarios (extensión, orientación, costo económico a solventar por el candidato, lugares de entrenamiento específicos muy escasos que actúan rigidizando el sistema, etc.).

b) La reciente política nacional (Plan de Especialistas para Chile), que involucra al Estado y a los Centros Formadores, debe profundizarse en un horizonte a mediano y largo plazo, para formar y distribuir especialistas. El estado debe sustentar y estimular una acción de fomento y no circunscribirse a la solución de coyunturas conflictivas urgentes.

c) Escasa contribución del sector privado de la salud en la formación de especialistas, siendo un sector muy beneficiado con la contratación de ellos.

d) Falta de un impulso global, como expresión de una política general y especial, para estimular y perfeccionar la vía de formación por adiestramiento en práctica, que a la fecha sólo depende del esfuerzo personal de cada médico interesado.

e) Las remuneraciones de los especialistas del sector público deben ser competitivas con las ofrecidas por el sector privado.

La cuantificación de la demanda de especialistas es compleja, y a su vez disímil por las variables concurrentes ya señaladas. La aproximación que hace al respecto el Ministerio de Salud se basa en información desglosada por patologías y áreas a lo largo de todo el país; fundamentalmente se realiza con indicadores como la relación entre el total de consultas por patología GES (Garantías Explícitas en Salud) y consultas no resueltas de ella en plazos determinados, o, la relación entre el total de cirugías no GES y el número de ellas no resueltas en un año. Estas tasas permiten construir un índice de satisfacción por patología y localidad. La autoridad considera aceptable un índice $<5$, de riesgo entre 5 y 10 y francamente crítico $>10$. Son muchas las regiones y las patologías que tienen índices inaceptables. En opinión del MINSAL estos vectores dan cuenta del $80 \%$ de la demanda de la población por especialistas y permiten ajustarla de acuerdo a áreas y especialidades.

Sin embargo, este método, que es una buena aproximación, tiene la limitante de considerar en el universo de pacientes sólo a los que solicitan consulta, sabiendo que hay mucha gente que desiste de hacerlo por no tener las facilidades para ello (comunicación, lejanía, locomoción, etc.), o no consulta por ausencia de síntomas, como ocurre en las enfermedades crónicas no trasmisibles, que requieren de un diagnóstico y tratamientos precoces, si se pretende disminuir sus complicaciones de grave impacto. En consecuencia, la situación real es de mayor detrimento que la sustentada por esos índices.

Parece atractivo dimensionar la demanda de especialistas en términos de horas de trabajo más que en número de especialistas con cargos rígidos. Definiciones de la necesidad de especialistas por millón de habitantes también permitirían enfoques de mayor resolución. Ello daría flexibilidad al sistema y respondería mejor a las demandas propias de áreas vecinas, que no pueden ser abordadas conjuntamente a pesar de su cercanía geográfica.

3. Vías de solución:

a) Mejorar substancialmente la atención primaria en el sector público, reforzando el enfoque familiar y comunitario, la prevención, la capacidad clínica básica y de interconsultas, para precisar diagnósticos, instalar tratamientos y dar curso a eventuales derivaciones de pacientes.

b) Potenciar la coordinación y cooperación entre el sistema asistencial público y el sistema universitario formador de especialistas.

c) Seguir ampliando en número y calidad los programas acreditados de especialistas cuyo eje es la atención secundaria: Medicina Interna, Medicina Familiar y de la Comunidad, Cirugía General, Pediatría, Siquiatría y Geriatría, de modo de respaldar y orientar la atención primaria y hacerla más resolutiva. Podría agregarse apoyo por parte de especialistas de disciplinas complejas de alta demanda que permitieran tamizar consultas y agilizar el sistema (ej. neurología, oftalmología, otorrinolaringología, dermatología).

d) Cualquier ampliación de los programas universitarios debe respetar la calidad de los mismos; dado que la supervisión docente del trabajo asistencial es la actividad más importante del proceso de formación de especialistas, la relación entre capacidad docente de un programa y su número de alumnos debe ser cuidadosamente evaluada y respetada. El financiamiento estatal de los programas debe hacerse cargo de la manutención de los candidatos (atendiendo a sus características familiares), la colegiatura y los gastos propios de la enseñanza, de modo que permita una jornada formativa exclusiva de 8 horas. Debe tenerse presente sin embargo, que aún una fuerte expansión de esos programas no sería capaz, en un plazo mediano de cubrir por sí sola las necesidades de especialistas del país.

e) En el caso de especialidades derivadas las universidades deben proponer programas más compactos y de menor duración, sin desmedro de la calidad, situación que ya está ocurriendo.

f) Los programas universitarios deben considerar la inclusión de competencias transversales 
como comunicación, administración y relación de ayuda. Ellos son contenidos necesarios para formar un buen especialista médico y hacerlo más eficiente y resolutivo.

g) Establecer la atención en red, creando en los CRS (Centro de Referencia de Salud) un nivel secundario verdaderamente resolutivo.

h) Entrenar a médicos de atención primaria, a través de programas ad hoc, en el uso de técnicas de alto rendimiento y resolución diagnóstica a desarrollar en centros primarios (ej. ecotomografía abdominal).

i) Los médicos de los programas universitarios con destinación conocida deben ser considerados empleados públicos y pagados consecuentemente por el Estado. Los documentos de resguardo de cumplimiento deben ser firmados entre los interesados y la autoridad estatal.

j) En áreas con falencias docentes a nivel nacional propiciar convenios de formación con universidades extranjeras cuya calidad debe ser acreditada por una instancia específica.

k) Propender a una mayor flexibilidad legal para que los médicos de atención diurna y de atención primaria puedan desarrollar una carrera funcionaria, y se facilite su movilidad dentro del servicio público sin interrupción de sus beneficios adquiridos.

I) Diseñar y aprobar una política nacional, para dar sustento, desarrollo y profundidad a la vía de adiestramiento en práctica (ver acápite siguiente). 4. La vía de adiestramiento en práctica:

La vía de certificación por adiestramiento en práctica es substancialmente diferente a la de programas universitarios; nació y se mantiene como respuesta a la falta de especialistas. Esta diferencia conceptual y metodológica hace necesario que el desarrollo de ambas vías sea independiente en cuanto a los servicios donde se asientan y los docentes o monitores a cargo. Los requisitos para optar a la certificación como especialista por la vía de adiestramiento en práctica son: a) haber trabajado al menos durante 5 años, en jornada de 22 horas semanales en un servicio acorde con la especialidad, donde existan al menos dos especialistas certificados, teniendo una ligazón contractual con este; aceptada la postulación el candidato debe aprobar un examen escrito, y luego un examen práctico.

CONACEM utiliza esta vía de reconocimiento de especialistas desde 1984, habiendo certificado a la fecha un total de 4.345 médicos, que, a su vez, constituyen el $34,6 \%$ del total de certificaciones. Para impulsar y desarrollar un gran Programa Nacional de Adiestramiento en Práctica es necesario mancomunar los esfuerzos de todos los estamentos interesados: MINSAL, Escuelas de Medicina, Colegio Médico de Chile AG., ASOCIMED y CONACEM. Cada cual, en su ámbito, deberá propender a hacer realidad o cooperar interactivamente en algunas de las siguientes medidas: a) Que los candidatos a los programas universitarios conozcan su destinación final en centros hospitalarios públicos desde el momento en que concursan, lugares donde deberían ser ubicados en parejas. Estos egresados deberán constituir el germen del cuerpo formador de médicos por adiestramiento en práctica en sus lugares de destino.

b) Contar con un catastro ajustado y actualizado de las necesidades locales o sectoriales de especialistas para optimizar las destinaciones. A su vez, se debe cuantificar selectivamente para cada especialidad, la capacidad formadora de los hospitales de la red de Salud para no caer en destinaciones espurias en lo docente.

c) El Ministerio de Salud debe comprometerse a dotar a esos lugares de destinación con la infraestructura, equipamiento e insumos, y del personal de apoyo médico necesario (enfermeras, matronas, tecnólogos, nutricionistas, etc.), que hagan posible el correcto desempeño del especialista.

d) Las destinaciones deben ser confluentes para distintas especialidades y así lograr polos de desarrollo y atracción regionales que constituyan grupos de trabajo que generen a mediano plazo centros de mediana y alta complejidad.

e) Favorecer la conectividad e intercambio entre varios centros regionales.

f) Apadrinar estos centros incipientes por Escuelas de Medicina con suficiente desarrollo para potenciarlos. Hay experiencias muy promisorias del pasado al respecto.

g) Pactar convenios de apoyo con universidades, incluso extranjeras, para el adiestramiento en práctica en áreas de mayor complejidad en algunas especialidades.

h) Diseñar una política de captación de candidatos propios de la zona, para iniciar su adiestramiento en práctica, con programas conocidos y preestablecidos.

i) Crear una política de recepción y acogida en cada zona que favorezca la radicación de los nuevos médicos, facilitando sus trámites de asentamiento familiar e incluyendo una asignación monetaria inicial transitoria.

j) Crear y desarrollar un programa integrado y progresivo de Educación Continua, que permita en períodos de 5 a 6 años reactualizar lo substantivo de cada especialidad. Esto es fundamental para estimular el desarrollo profesional de los especialistas y su permanencia en la zona.

k) El Ministerio de Salud debe normar para hacer efectivas facilidades para asistir a congresos o cursos que correspondan al proceso de enseñanza continua de la especialidad.

I) Definir remuneraciones competitivas, progresivamente estimulantes, con asignaciones por competencia, estímulo, jornadas prioritarias, e incorporación a la carrera funcionaria. 
m) Establecer comunicación electrónica en reentre los diversos estamentos de atención, primario, secundario y terciario para agilizar la atención y determinar correctamente las derivaciones.

n) Mejorar substancialmente la comunicación entre el Ministerio de Salud y los actores locales.

o) Control de la calidad para lograr la certificación y luego recertificación de los médicos en adiestramiento en práctica.

\section{Considerandos finales:}

La complejidad y extensión del déficit de especialistas en Chile hace necesario seguir avanzando en la política que la autoridad ministerial está actualmente desarrollando con un significativo aumento de los recursos presupuestarios destinados a la formación de especialistas. Este esfuerzo demanda una profundización de la coordinación de todos los actores de la Medicina Chilena, en beneficio de nuestros pacientes. 